\title{
THE LATTICE THEORETIC PART OF TOPOLOGICAL SEPARATION PROPERTIES
}

\author{
by H. SIMMONS \\ (Received 2nd September 1976)
}

In this paper we show that for each $n \in\{2,3,4,5\}$ the topological separation property $T_{n}$ can be decomposed

$$
T_{n}=T_{0}+C+N_{n}
$$

where $C, N_{2}, \ldots, N_{n}$ are purely lattice theoretic properties with the expected implications holding between them.

The property $C$ is discussed briefly in $\S 1$ where it is explained that $C$ is the lattice theoretic analogue of ring theoretic semisimplicity and also related to the topological property $T_{1}$. The four properties $N_{2}, \ldots, N_{5}$ are discussed in $\$ 2$. The properties $N_{4}, N_{5}$ are the lattice theoretic analogues of topological normality and complete normality. For this reason we call $N_{2}, \ldots, N_{5}$ normality properties. In $\$ 3$ we establish the above decomposition and show that $C+N_{n}$ can be thought of as the lattice theoretic part of $T_{n}$. In particular we show that a space has $C+N_{n}$ if and only if its open set lattice is isomorphic to the open set lattice of a $T_{n}$ space. These results extend the remarks of Davis $(1, \$ 3)$. Some of these results are also related to results of (2) and (3). Property $C+N_{3}$ is the lattice theoretic analogue of topological regularity, and so we call $C+N_{2}, \ldots, C+N_{5}$ regularity properties. Finally in $\$ 4$ we say a few words about the $T_{1}$ case.

\section{Conjunctivity}

Throughout we are concerned with distributive lattices with a top 1 and a bottom 0 such that $0 \neq 1$. Consequently we use the word 'lattice' in this restricted sense. Each topological space $S$ gives us a lattice, namely the lattice $O(S)$ of open sets of $S$. Such a lattice has certain extra properties (in the terminology of (2) it is a frame), however we will not use these extra properties.

Definition 1. A lattice $L$ has property $C$, or is conjunctive, if for each two elements $a, b$ of $L$ with $a \neq b$, there is an element $z$ of $L$ such that $a \vee z=1, b \vee z \neq 1$.

An easy application of Zorn's lemma shows that two elements of a lattice are equal when they belong to exactly the same prime ideals of the lattice. Thus (by analogy with the ring case) we may say that each lattice is semiprime. It is fairly easy to verify that conjunctive lattices are the analogues of semisimple rings, that is a 
lattice is conjunctive if and only if two elements of the lattice are equal when they belong to exactly the same maximal ideals. For this reason it seems plausible that conjunctivity will have some lattice theoretic importance. In fact conjunctivity (or rather its dual, disjunctivity) has been used in purely lattice theoretic work.

In this paper we are interested in conjunctivity for a different reason, namely for its topological significance. The following result is proved in (3, Theorem 4).

Lemma 2. For each space $S$ the following are equivalent.

(i) The lattice $O(S)$ has $C$.

(ii) For each point $p$ and open set $A$ of $S$ with $p \in A$, there is a point $q$ of $S$ such that $\{q\}^{-} \subseteq A \cap\{p\}^{-}$.

Notice that for each $T_{1}$ space $S$ the lattice $O(S)$ has $C$ (since for each point $p$ of such a space, $\{p\}^{-}=\{p\}$ ). Thus each $T_{1}$ space has $T_{0}+C$, however (as is pointed out in (3)) $T_{1}$ is stronger than $T_{0}+C$. In this paper we show that for many purposes the separation property $T_{1}$ can be replaced by $T_{0}+C$.

\section{Normality properties}

The following definition is four definitions in one and should be read as such.

Definition 3. A lattice $L$ has property $N_{2}$, or $N_{3}$, or $N_{4}$, or $N_{5}$ (respectively) if for each two elements $a, b$ of $L$ with

$$
\begin{aligned}
& \text { (2) } a \vee b=1, b \neq 1, a \neq 1, \\
& \text { or (3) } a \vee b=1, b \neq 1, \\
& \text { or (4) } a \vee b=1, \\
& \text { or (5) [no restrictions] }
\end{aligned}
$$

there are elements $x, y$ of $L$ such that $x \wedge y=0$ and

$$
\begin{aligned}
& \text { (2) } x \neq a, y \neq b \\
& \text { or (3) } a \vee x=1, y \neq b, \\
& \text { or (4) } a \vee x=1, b \vee y=1, \\
& \text { or (5) } x \leqslant b \leqslant a \vee x, y \leqslant a \leqslant b \vee y,
\end{aligned}
$$

respectively.

The following lemma is easily verified.

Lemma 4. The sequence of properties $N_{2}, \ldots, N_{5}$ is increasing in strength, that is $N_{5} \Rightarrow N_{4}, N_{4} \Rightarrow N_{3}, N_{3} \Rightarrow N_{2}$.

Notice that in Definition 3 each of the restrictions $(3,4)$ on the pair $a, b$ can be replaced by the restriction (2).

As with conjunctivity these four properties have topological significance. Part 5 of the next theorem is due to D. Macnab. 
Theorem 5. For each space $S$ and each $n \in\{2,3,4,5\}$ the conditions $(n, i),(n, i i)$ given below are equivalent.

2. (i) The lattice $O(S)$ has $N_{2}$.

(ii) For each two points $p, q$ of $S$ with $\{p\}^{-} \cap\{q\}^{-}=\emptyset$, there are disjoint open sets $X, Y$ of $S$ such that $p \in X, q \in Y$.

3. (i) The lattice $O(S)$ has $N_{3}$.

(ii) For each point $p$ and open set $A$ of $S$ with $\{p\}^{-} \subseteq A$, there are open sets $X, Y$ of $S$ such that $p \in Y \subseteq X^{\prime} \subseteq A$.

4. (i) The lattice $O(S)$ has $N_{4}$.

(ii) The space $S$ is normal.

5. (i) The lattice $O(S)$ has $N_{5}$.

(ii) The space $S$ is completely normal.

Proof. 2. (i) $\Rightarrow$ (ii). Suppose $O(S)$ has $N_{2}$ and consider any two points $p, q$ of $S$ with $\{p\}^{-} \cap\{q\}^{-}=\emptyset$. Let $A=\{p\}^{-\prime}, B=\{q\}^{-\prime}$ so that $A, B$ are elements of $O(S)$ with

$$
A \cup B=S, \quad B \neq S, \quad A \neq S .
$$

Since $O(S)$ has $N_{2}$ there are open sets $X, Y$ of $S$ such that

$$
X \cap Y=\emptyset, \quad X \nsubseteq \subset A, \quad Y \nsubseteq B
$$

which easily translates into the required result.

2. (ii) $\Rightarrow$ (i). Suppose that $S$ has the separation property of (ii) and consider any open sets $A, B$ of $S$ such that (a) holds. Then $A^{\prime}, B^{\prime}$ are disjoint non-empty closed sets so, with $p \in A^{\prime} q \in B^{\prime}$, we have $\{p\}^{-} \cap\{q\}^{-}=\emptyset$. The separation property (ii) now gives us disjoint open neighbourhoods $X, Y$ of $p, q$ hence (with this pair $X, Y$ ) we easily verify $(b)$, which is the required result.

3. (i) $\Rightarrow$ (ii). Suppose that $O(S)$ has $N_{3}$ and consider any point $p$ and open set $A$ of $S$ with $\{p\}^{-} \subseteq A$. Let $B=\{p\}^{-\prime}$ so that $A, B$ are elements of $O(S)$ with

$$
A \cup B=S, \quad B \neq S
$$

Since $O(S)$ has $N_{3}$ there are open sets $X, Y$ of $S$ such that

$$
X \cap Y=\emptyset, \quad A \cup X=S, \quad Y \not \subset B
$$

which gives the required result

$$
p \in Y \subseteq X^{\prime} \subseteq A .
$$

3. (ii) $\Rightarrow$ (i). Suppose that $S$ has the separation property of (ii) and consider any open sets $A, B$ of $S$ such that (c) hold. There is some point $p \in A-B$ so (ii) gives us open sets $X, Y$ such that (e) holds. But then, since $p \in B^{\prime}$, (e) gives us (d), which is the required result.

4. This is trivial since $N_{4}$ is a direct translation of topological normality.

5. (i) $\Rightarrow$ (ii). Suppose that $O(S)$ has $N_{5}$ and consider any two subsets $H, K$ of $S$ such that

$$
H \cap K^{-}=H^{-} \cap K=\emptyset .
$$

(We must produce disjoint open sets $X, Y$ of $S$ such that $H \subseteq X, K \subseteq Y$.) 
Let $A=H^{-\prime}, B=K^{-\prime}$ so that

$$
H \subseteq A^{\prime} \cap B, \quad K \subseteq A \cap B^{\prime}
$$

The $N_{S}$ property of $O(S)$ gives us disjoint open sets $X, Y$ of $S$ such that

$$
X \subseteq B \subseteq A \cup X, \quad Y \subseteq A \subseteq B \cup Y .
$$

But then (remembering that $X \cap Y=\emptyset$ ) we have

$$
\begin{aligned}
A^{\prime} \cap B & \subseteq A^{\prime} \cap(A \cup X) \\
& =A^{\prime} \cap X \\
& \subseteq Y^{\prime} \cap X \\
& =X
\end{aligned}
$$

and similarly

$$
A \cap B^{\prime} \subseteq Y
$$

which verifies (ii).

5. (ii) $\Rightarrow$ (i). Suppose that $S$ is completely normal and consider any open sets $A, B$ of $S$. Let

$$
H=A^{\prime} \cap A^{-} \cap B, \quad K=A \cap B^{\prime} \cap B^{-}
$$

so that $H^{-} \subseteq A^{\prime}, K^{-} \subseteq B^{\prime}$, and hence ( $f$ ) holds. The complete normality of $S$ now gives us open sets $U, V$ of $S$ such that

$$
U \cap V=\emptyset, \quad H \subseteq U, \quad K \subseteq V .
$$

Let

$$
X=\left(A^{-\prime} \cup U\right) \cap B, \quad Y=\left(B^{-\prime} \cup V\right) \cap A
$$

so that $X, Y$ are open, $X \subseteq B, Y \subseteq A$, and we easily check that $X, Y$ are disjoint. For each set $W$ we have

$$
W=W^{-} \cap\left(W \cup W^{-\prime}\right)
$$

so that

$$
\begin{aligned}
A^{\prime} \cap B & =\left(A^{-\prime} \cup\left(A^{\prime} \cap A^{-}\right)\right) \cap B \\
& =\left(A^{-\prime} \cap B\right) \cup\left(A^{\prime} \cap A^{-} \cap B\right) \\
& =\left(A^{-\prime} \cap B\right) \cup H \\
& \subseteq\left(A^{-\prime} \cap B\right) \cup(U \cap B) \\
& =\left(A^{-\prime} \cup U\right) \cap B \\
& =X
\end{aligned}
$$

and hence $B \subseteq A \cup X$. Similarly $A \subseteq B \cup Y$, which verifies (g), as required.

A corollary of this theorem is that the definition

$$
T_{n}=T_{1}+N_{n}
$$

(for $n=4,5$ ) also holds for the cases $n=2,3$. In the next section we show that for all four cases $T_{1}$ can be weakened to $T_{0}+C$. 


\section{Regularity properties}

In this section we discuss the four properties $C+N_{n}$ (for $n \in\{2,3,4,5\}$ ). Again these properties have topological significance although only $C+N_{2}, C+N_{3}$ are given explicit topological characterisations.

First we prove three lemmas.

Lemma 6. For each space $S$ the following are equivalent.

(i) The lattice $O(S)$ has $C+N_{2}$.

(ii) For each two points $p, q$ of $S$ with $\{p\}^{-} \neq\{q\}^{-}$, there are disjoint open sets $X, Y$ of $S$ such that $p \in X, q \in Y$.

(iii) For each two points $p, q$ of $S$ with $\{p\}^{-} \neq\{q\}^{-}$, there are disjoint open sets $X, Y$ of $S$ such that $\{p\}^{-} \subseteq X,\{q\}^{-} \subseteq Y$.

Proof. (i) $\Rightarrow$ (ii). Suppose that $O(S)$ has $C+N_{2}$ and consider any two points $p, q$ of $S$ with $\{p\}^{-} \neq\{q\}^{-}$. Then either $p \in\{q\}^{-\prime}$ or $q \in\{p\}^{-\prime}$. By symmetry we may assume that $p \in A$, where $A=\{q\}^{-1}$.

Now $O(S)$ has $C$ so Lemma 2 gives us some point $r$ of $S$ with $\{r\}^{-} \subseteq A \cap\{p\}^{-}$. In particular $\{r\}^{-} \cap\{q\}^{-}=\emptyset$ so that, since $O(S)$ has $N_{2}$, Theorem $5(2)$ gives us disjoint open sets $X, Y$ such that $r \in X, q \in Y$. But $r \in\{p\}^{-}$so that $p \in X$, which gives us (ii).

(ii) $\Rightarrow$ (iii). Suppose that (ii) holds, so that for each two points $p, q$ of $S$

$$
\{p\}^{-} \neq\{q\}^{-} \Rightarrow\{p\}^{-} \cap\{q\}^{-}=\emptyset \text {. }
$$

Hence, for each two points $p, r$ of $S$

$$
r \in\{p\}^{-} \Rightarrow\{r\}^{-}=\{p\}^{-} \text {. }
$$

Using this observation we easily deduce (iii).

(iii) $\Rightarrow$ (i). This follows easily from Lemma 2 and Theorem 5(2).

Lemma 7. For each lattice $L$ the following are equivalent.

(i) L has $\mathrm{C}+\mathrm{N}_{3}$.

(ii) For each two elements $a, b$ of $L$ with $a \neq b$, there are elements $x, y$ of $L$ such that $x \wedge y=0, a \vee x=1, y \neq b$.

Proof. (i) $\Rightarrow$ (ii). Suppose that $L$ has $C+N_{3}$ and consider any two elements $a, b$ of $L$ with $a \neq b$. Since $L$ has $C$ there is an element $z$ of $L$ such that

$$
a \vee z=1, \quad b \vee z \neq 1,
$$

in particular $a \vee b \vee z=1$. But $L$ has $N_{3}$ so there are elements $x, y$ of $L$ with

$$
x \wedge y=0, \quad a \vee x=1, \quad y \neq b \vee z .
$$

In particular $y \neq b$, and so we have verified (ii).

(ii) $\Rightarrow$ (i). Suppose that (ii) holds.

Firstly consider any two elements $a, b$ of $L$ with $a \neq b$. Let $x, y$ be the elements given by (ii) and consider $z=x$. Then $a \vee z=1$, and $b \vee z \neq 1$, for otherwise 


$$
\begin{aligned}
b \wedge y & =(b \wedge y) \vee(x \wedge y) \\
& =(b \vee x) \wedge y \\
& =1 \wedge y \\
& =y
\end{aligned}
$$

so that $y \leqslant b$. This shows that $L$ has $C$.

Secondly consider any two elements $a, b$ of $L$ with $a \vee b=1, b \neq 1$. Then $a * b$ (for otherwise $b=a \vee b=1$ ) so (ii) gives us the required elements $x, y$ to verify that $L$ has $N_{3}$.

This completes the proof of the lemma.

Using this lemma we can easily deduce the following lemma.

Lemma 8. For each space $S$ the following are equivalent.

(i) The lattice $O(S)$ has $C+N_{3}$.

(ii) The space $S$ is regular.

We now come to the two theorems which, in some way, justify our claim that $C+N_{n}$ is the lattice theoretic part of the $T_{n}$ separation property. The case $n=4$ of the first of these theorems is essentially the main result of (3).

Theorem 9. For each $T_{0}$ space $S$ and each $n \in\{2,3,4,5\}$ the following are equivalent.

(i) The lattice $O(S)$ has $C+N_{n}$.

(ii) The space $S$ is $T_{n}$.

Proof. (i) $\Rightarrow$ (ii). Suppose that (i) holds so that (since for each two points $p, q$ of the $T_{0}$ space $S$,

$$
p=q \Leftrightarrow\{p\}^{-}=\{q\}^{-} \text {) }
$$

Lemmas 4, 6 show that $S$ is $T_{2}$. This verifies (ii) for the case $n=2$. But now the case $n=3$ follows by Lemma 8 , and the cases $n=4,5$ follow by Theorems 5(4), 5(5) respectively.

(ii) $\Rightarrow$ (i). Suppose that (ii) holds, in particular $S$ is $T_{1}$. Then (i) follows by Lemma 2 and Theorem 5.

Before we prove the next theorem we need some terminology and notation.

We say two spaces $S, T$ are isomorphic if their open set lattices $O(S), O(T)$ are isomorphic (as lattices). Notice that if $S, T$ are homeomorphic then $S, T$ are isomorphic, (for if $f: S \rightarrow T$ is a homeomorphism then

$$
\begin{gathered}
O(T) \rightarrow O(S) \\
V \mapsto f^{-}[V]
\end{gathered}
$$

is a lattice isomorphism). However there are isomorphic spaces which are not homeomorphic. (This phenomenon is concerned with the sobriety of the spaces.) It seems reasonable to say that a property of spaces is lattice theoretic if it depends only on the isomorphism type of the space. 
For each space $S$ let $S^{\wedge}$ be the $T_{0}$-corrected version of $S$. Thus we put

$$
S=\left\{\{p\}^{-}: p \in S\right\}
$$

and topologize $S^{\wedge}$ by

$$
O\left(S^{\wedge}\right)=\left\{U^{\wedge}: U \in O(S)\right\}
$$

where, for $U \in O(S)$,

$$
U^{\wedge}=\left\{\{p\}^{-}: p \in U\right\} .
$$

We easily check that for each $p \in S, U \in O(S)$,

$$
\{p\}^{-} \in U^{\wedge} \Leftrightarrow p \in U .
$$

Notice also that $S^{\wedge}$ is $T_{0}$,

$$
\begin{gathered}
S \rightarrow S^{\wedge} \\
p \mapsto\{p\}^{-}
\end{gathered}
$$

is a continuous open surjection, and

$$
\begin{gathered}
O(S) \rightarrow O\left(S^{\wedge}\right) \\
U \mapsto U^{\wedge}
\end{gathered}
$$

is an isomorphism. In particular $S$ is isomorphic with the $T_{0}$ space $S^{\wedge}$. Clearly if $S$ itself is $T_{0}$ then $S=S^{\wedge}$.

Theorem 10. For each space $S$ and $n \in\{2,3,4,5\}$ the following a re equivalent.

(i) $S$ is isomorphic with a $T_{n}$ space.

(ii) The lattice $O(S)$ has $C+N_{n}$.

(iii) $S^{\wedge}$ is $T_{n}$.

Proof. (i) $\Rightarrow$ (ii). Suppose $T$ is a $T_{n}$ space such that $O(S), O(T)$ are isomorphic. Then, by Theorem $9, O(T)$ has $C+N_{n}$, hence so also does $O(S)$, as required.

(ii) $\Rightarrow$ (iii). This follows by Theorem 9 since $S, S^{\wedge}$ are isomorphic and $S^{\wedge}$ is $T_{0}$.

(iii) $\Rightarrow$ (i). This follows since $S, S^{\wedge}$ are isomorphic.

\section{The $T_{1}$ case}

It would be nice if we could find a lattice theoretic property $N_{1}$ such that $N_{2} \Rightarrow N_{1}$ and each of Theorems $5,9,10$ could be extended to the case $n=1$. However it appears that no such property exists.

A reading of (1, Theorem 2) suggests that Theorem 10 can be extended by the following.

Theorem 11. For each space $S$ the following are equivalent.

(i) $S$ is isomorphic with a $T_{1}$ space.

(ii) For each point $p$ and open set $A$ of $S$, if $p \in A$ then $\{p\}^{-} \subseteq A$.

(iii) $S^{\wedge}$ is $T_{1}$. 
In fact we can easily verify that (ii) $\Leftrightarrow$ (iii) and (iii) $\Rightarrow$ (i), but, as pointed out in (3), (i) $\Rightarrow$ (ii) is false.

It could be that $T_{0}$ is not the correct basic separation property to use. For there are isomorphic, non-homeomorphic $T_{0}$ spaces. Perhaps the stronger separtation property of sobriety should be used (for isomorphic sober spaces are homeomorphic). Notice that Theorem 10 still holds if $S^{\wedge}$ is interpreted as the sobering up of $S$ (rather than the $T_{0}$-correction of $S$ ). This is because the sobering up of $S$ is again isomorphic with $S$ (and is $T_{0}$ ). If we take this point of view then it is not reasonable to extend the above results to cover the $T_{1}$ case, for sobriety and the $T_{1}$ property are incomparable.

\section{REFERENCES}

(1) A. S. Davis, Indexed systems of neighborhoods for general topological spaces, Amer. Math. Monthly 68 (1961), 886-893.

(2) C. H. DowkER and DONA PAPERT STRAuss, Separation axioms for frames, Topics in Topology, Ed. by Á. Császár (North Holland, 1972), 223-240.

(3) D. S. MACNAB and H. Simmons, Conjunctive topological spaces.

\section{UNIVERSITY OF ABERDEEN}

